I CRI -

PREPRINT

C.NGF-SUCI2L - 3




SUPERNOVA MODELS AND LIGHT CURVES*

Thomas A. Weaver

Lawrence Iivermore Laboratory

Livermore, CA 94550

\author{
S. E. Woosley ${ }^{\dagger}$ \\ University of California, Senta Cruz \\ Sante Cruz, CA 95064 \\ and \\ Lawrence Livermore Laboratory \\ Livermore, CA 94550
}

\title{
ABSTRACT
}

This talk briefly reviews the current status of our understanding of Type II supernovae with particular emphasis on the processes responsible for the emission of electromagnetic radiation. In addition, a relatively novel evolutionary scenario that appears to lead to a Type I supernova explosion is presented.

\section{TYPE II SUPERNOVAE}

Type II supernova have long been associated with messive stars with extended hydrogen envelopes. This association is due both to direct spectroscopic evidence $1,2,3$ and to the quite distinct correlation of Iype II supernovere with the spiral arms of galaxies ${ }^{4}$, suggesting that their progenitors are bright, short-1ived $(<30$ million years) $O$ and/or B stars with mass $\geqslant 10 \mathrm{M}$.

We ${ }^{5-8}$ have approached this issue from the theoretical viewpoint of evolving massive stars from the zero-age main sequence, through their various hydrostatic and explosive nuclear burning phases, and then comparing the characteristics of the supernove explosions that result to observations. Our numerical model ${ }^{5}$ of these events incorporates implicit hydrodynamics with time-dependent convection and semiconvection, and a careful treatment of the complex nuclesr processes that characterize the advanced nuclear burning stages. Spherical symmetry, and thus the absence of rotation and megnetic fields, is assumed, as is the unimportance of mass loss.

Complete evolutionary calculations have been performed for 15 and $25 \mathrm{M}$. Population I stars, and have been sumarized by Weaver and Woosley ${ }^{6}$. (See Ref. 5, 6, and 7 for a discussion of the relation of these cBlculations to previous work.) We shall concentrate in this talk on the observale effects of the explosive death of these stars.

*Work performed under the auspices of the U.S. Department of Energy by LIVL under contract number W-7405-ENG-48.

tWork performed in part under ISF contract number AST-79-09102. 


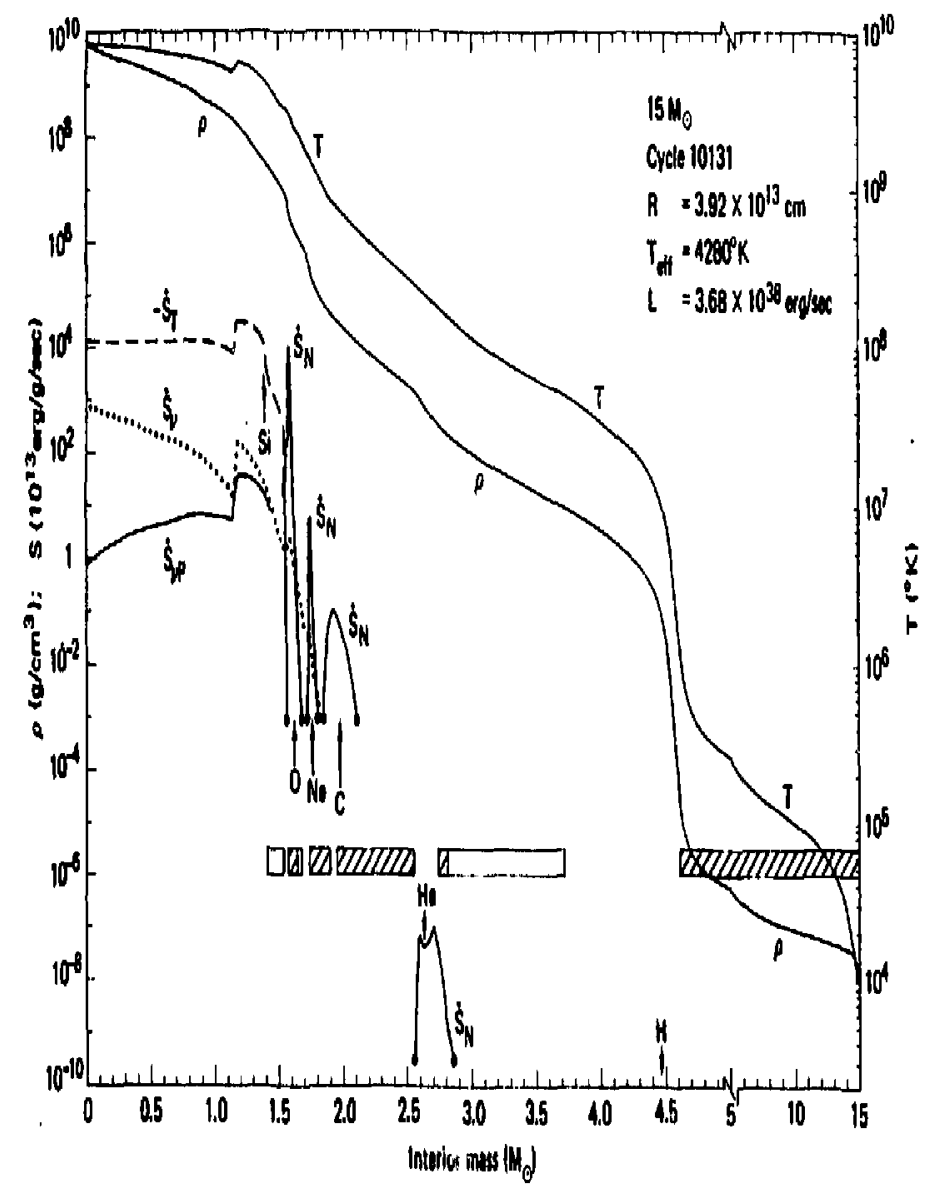

Pig. 1 The thermodynamic structure of the presupernova $15 \mathrm{M}$ model star is shown as a function of mass fraction. 5 Note that the density and temperature profilles are plotted such that the curves vill maintain - coustant separation for the case $\rho$ a $\mathbb{T}^{3}$. Here $-S_{\mathrm{T}}$ is the total local energy loss rate due to both neutrino emission and nuclear photodisintegration, $\dot{S}$ is the total neutrino energy loss rate, and $\dot{S}$ is the neutrino energy loss rate due to the thermal plesna processes. The nuclear enerwy generation rate profiles for the various nuclear burning shells are labelled $\dot{s}_{y}$, and the principal nuclear fuel is Indicated. All energy generation and loss rates share the connon scale denoted by "\$". Aetive convective regions are indicated by striped bars, wlile seni-convective and convectively neutral reglons are shown as outlined bars. In this figure, $R_{1} \mathbb{T}_{\text {eff }}$, and $\mathrm{L}$ denote the photospheric radius, effective temperature, and optical luninosity, respectivifly. 


\section{PRESUPERNOVA EVOLUTTON}

We find that the massive stars we have studied gradually deplete their store of nuclear fuel by burning theje initially predominantly hydrogen composition successively into helium, carbon, neon, oxygen, silicon and finally iron. In general, each successive fuel is ignited in the star's core, while shells of the lighter elements remain in the legion outside the core, usually separsted by active nuclear burning shells. These burning shells are typically convective and are separated by sufficiently steep entropy gradients so that mixing between shells does not occur. The collapse of the core is triggered by the endothermic photodisintegration of iron ${ }^{5}$ (c.f. Burbidge, Burbidge, Fowler, and Hoyle ${ }^{9}$. At this stage, about $2 / 3$ of the star's mass resides in a tenuous red-supergiant envelope composed mostly of hydrogen $(-60 \%)$ and helium ( $-40 \%)$, and having nearly constant $10^{-} \mathrm{Bg} \mathrm{cm}^{-1}$ density and $10^{5} \mathrm{~K}$ temperature (see $\mathrm{Fig} .1$ and Ref. 5). The radii of the 15 and $25 \mathrm{~N}_{0}$ stars are respectively 4 and $7 \times 10^{13} \mathrm{~cm}$.

\section{SHOCK WAVE PROPAGATION}

The outgoing shock wave that is thought to result from the collapse and bounce of the core (c.f. Wilson ${ }^{10}$ ) can then (if sufficiently strongl eject the mentle and envelope of the star, while the core of the ster recollapses to form a neutron star or black hole. This shock also induces explosive nuclear burning in the region just above the core, which though crucial to the synthesis of the elements in stars, adds only about 10 to $20 \%$ to the energy of the final supernova explosion. Much of the silicon layer directly over the iron core reaches temperatures above 4 billion degrees and is burned into 0.1 to $0.4 \mathrm{M}_{0}$ of radioactive $56_{\mathrm{Ni}}$ (the most strongly bound nucleus that can be formed from the nearly equal numbers of neutrons and protons present in the initial fuel). '56Ni, however, beta decays first to ${ }^{56}$ Co and then to ${ }^{56} \mathrm{Fe}$ with a half-life of 6.1 and 78.5 days, respectively. The $\gamma$-rays and positrons that result from these decays represent an important late-time input to the thermal energy of the exploding star.

As the shock wave continues to propagate out through the mantle $\left(0 \geqslant 10 \mathrm{~g} \mathrm{~cm}^{-1}\right)$ of the star, the energs needed to heat and accelerate each successive mass shell' is smoothly derived from the subsonic deceleration and adiabatic decompression of the underlying material. Sensitivity tesis ${ }^{6}$ show that except in the immediate vicinity of the mass cut between the mantle and the collapsed core, only the observable final energy of the outward-going shock wave and not the details of its formation are important in determining postshock conditions for a given presupernova configuration. The entropy of the postshock material is roughly constant due to the approximate cancellation of the centrally depressed (by neutrino emission) presupernova entropy profile by the centrally peaked profile of entropy production by the shock (due to shock deceleration). Since sufficient 
time is available for pressure belance to be established, this constant entropy profile results in nearly constant density and temperature profiles behind the shock front--in contrast to the thin, high density shells formed by a strong point explosion in an initially constant density medium. 11



Fig. 2 Velocity as a function of interior mass coordinate in the $1.3 \times 10^{51} \mathrm{erg}, 15 \mathrm{M}_{0}$ supernova model at, and fust after, the breakout of the shock wave from the surface. Each curve is labeled with the time in days since core collapse, and dotted line segments indicate regions with conditions allowing the growth of RayleighTaylor instabilities.

Figure 2 details the hydrodynamical behavior of the mantle and envelope of our $15 \mathrm{M}_{0}$ model star undergoing a $1.3 \times 10^{51}$ erg explosion (Model 15A, see Ref. 6). We shall discuss this case below as a typical Type II supernove because of its concordance with observation, and because $15 M_{0}$ stars are observed to be tore numerous than $25 M_{0}$ stars ${ }^{12}$, which we find to have generally similar behevior. During the time $t=60-500 \mathrm{sec}$ (measured from core collapse) the shock rave reaches and accelerates down the steep, 6-order-of-magnitude density gradient at the edge of the mantle, while the postshock material adiabatically expands and cools, initially unhindered by the surrounding lor density envelope. By the time the exploding mantle has swept up sufficient envelope material to be significantly decelerated ( $t-3,000 \mathrm{sec}$ ), its density has dropped to below $10^{-3}$ $\mathrm{g} \mathrm{cm}{ }^{-3}$ and 1 ts temperature below $2 \times 10^{6} \mathrm{~K}$. Under these conditions the expangion is hypersonic so that the deceleration of the mantle by the envelope is mediated by a reverse shock wave (see also Ref. 13). 
This reverse shock has almost reached the center of the star when the principal shock wave raches and accelerates down the final density gradient at the eage of the star at $t=1.1$ days. Subsequent adiabatic excansion over the next few days leads to the final velocity profile shown in Figure 2. Note that the relocity of the mantle has been reduced to below $1000 \mathrm{~km} / \mathrm{s}$ both by the action of the reverse shock and the restraining pressure exerted by the decompessing envelope. The mantle, which contains virtually all the $Z>2$ elements synthesized by the star, including the $56 \mathrm{Ni}$, is thus left with only about $5 \%$ of total energy of the explosion.

Density and temperature profiles at, and just after, shock breakout are shown in Figures 3 and 4 , and regions with conditions allowing the growth of Rayleigh-Taylor instabilities are indicated. In contrast to the more ad-hoc models studiea by Chevalier and Kleln 14 , we find that most of the envelope is not subject to RayleighTaylor instabilities during its acceleration. As pointed out by Lasher 15 , however, the presence of such instabilities is likely to be a sensitive function of the density gradient in the convective redgiant envelope, and thus of our still incomplete understanding of superadiabatic convection.

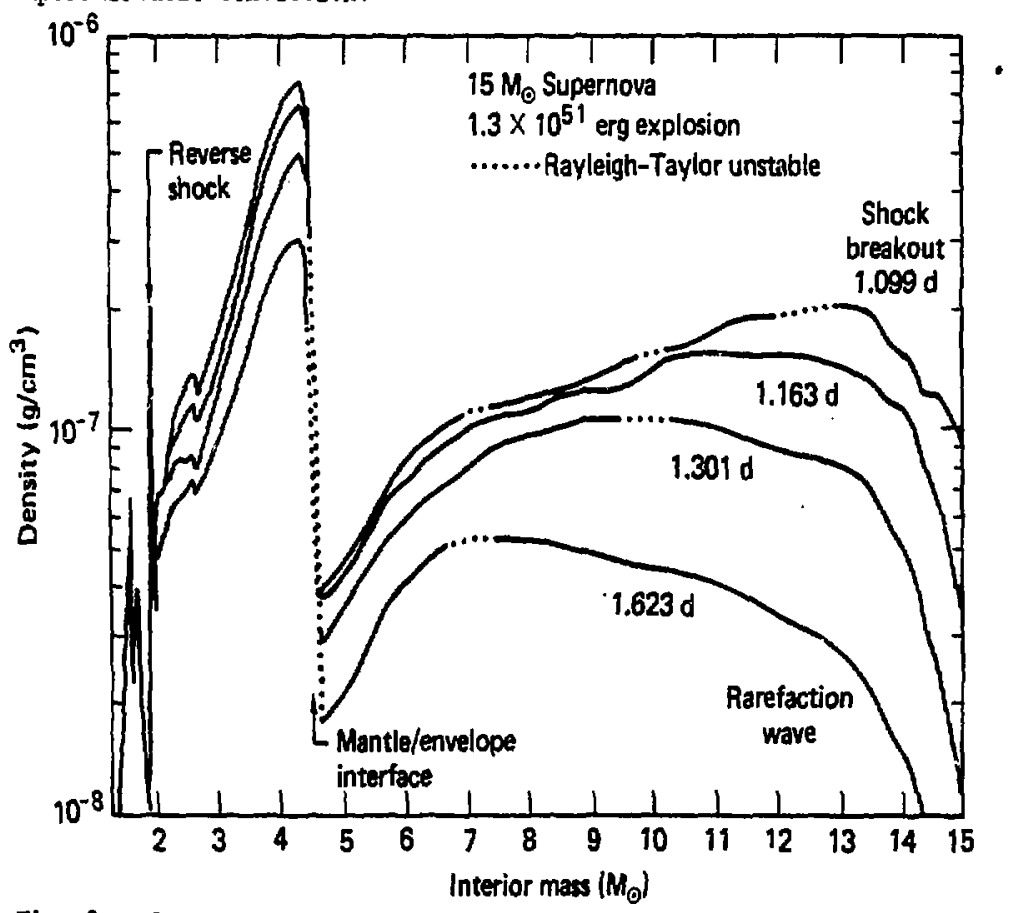

Fig. 3 Density as a function of interior mass coordinate in the $1.3 \times 10^{51} \mathrm{erg}, 15 \mathrm{M}$ supernors model at, and fust after, the breakout of the shock weve from the surface. Each curve is labeled with the time in days since core collapse, and dotted line segments indicate regions with conditions allowing the growth of RayleighTaylor instabilities. 


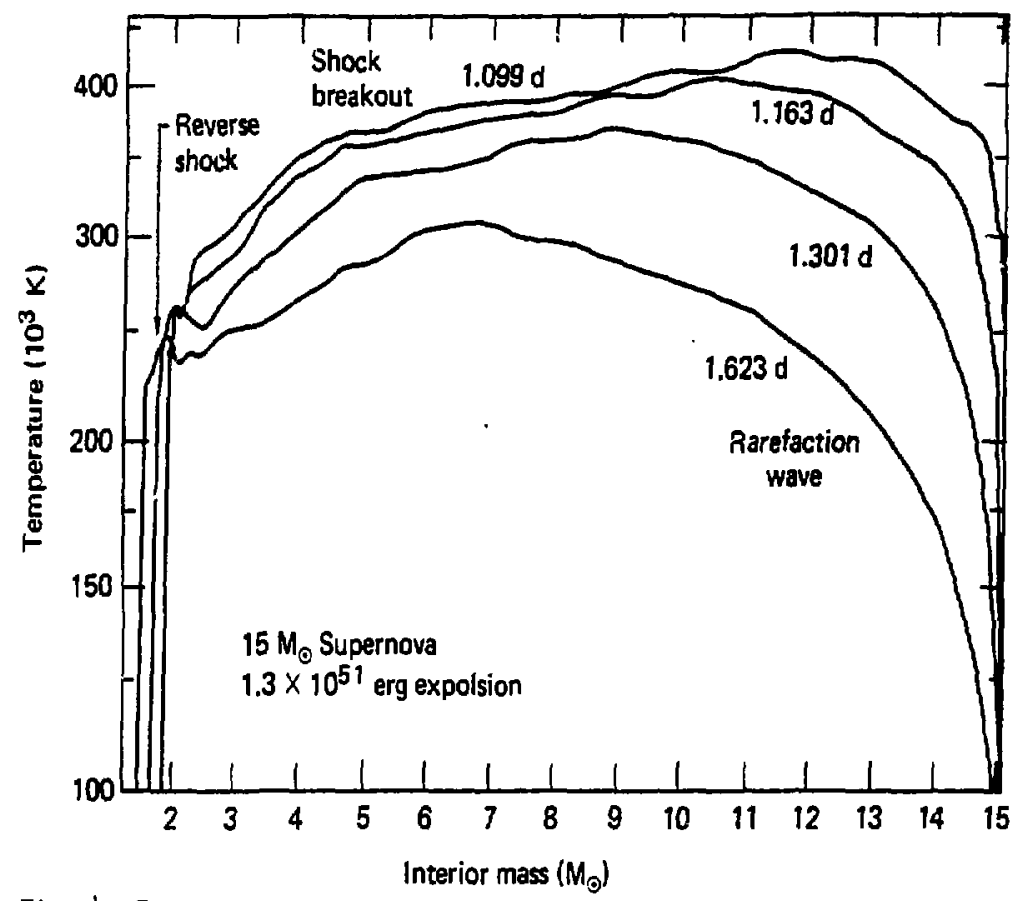

Fig. I Temperature profiles corresponding to the density profiles shown in Fig. 3.

\section{TYPE II SUPERNOVA LIGHT CURVES}

The light curves produced by two $15 \mathrm{M}_{0}$ supernova models (with differing explosion energies of 1.3 and $3.3 \times 10^{51}$ ergs due to differing assumptions about detailed core physics) are shown in Figure 5, compared to photometric data for Supernova $1969 \mathrm{l}$ in NGC 1058, perhaps the best observed Type II supernova. 2,16 SN 19692 is characteristic of a comon subcless of Type II supernovae which shows a 2-3 month initial plateau in its visual emission $\left(M_{\mathrm{v}}=-17\right)$ followed by a rapid decline of about 2 stellar magnitudes and then a slower decline at a rate of -3 magnitudes/year. (SN $1970 \mathrm{~g}$ in NGC $5457=\mathrm{MnOI}$ is another recent example of this subclass). 17 It is apparent that the theoretical and observational results are in excellent agreement. As Figure 4 shows, an increase in the energy of the core explosion produces a roughly linear increase in the optical brightness together with a shorter "plateau". The results for our $25 \mathrm{M}_{0}$ models are generally quite similar. Note in particular that this agreement has not been achieved by normalizing the observational absolute magnitude so as to provide the best fit.

Figures 6 and 7 compare derived observational SN $1969 \ell$ results $2,16,18$ for the temperature and radius of the supernova photosphere with the corresponding theoretical results. Figure 8 shows a comparison between observed absorption line velocities and photospheric 
$\operatorname{Tim}, \mathrm{d} y$



F1g. 5 Light curve of Mype II supernova $1969 \mathrm{l}$ compared to $15 \mathrm{M}$ theoretical sodels, ${ }^{6}$ Here, $\mathrm{H}_{\mathrm{o}}$ is the absolute holometric magnitude of the supernova and time is measured vith respect to core bounce for the theoretical models and Julian dey 2,440,560 for the observations. The solid and dotted lines give the bolonetric luminosity for the $15 \mathrm{Mg}$ models (15A and 158), while the data points for 1969h are either derived from the UBP deta of Ciatti, et al. 16 as transformed by the method of Schurmann, et al. ${ }^{21}$ (open circles) or obtained by integrating the multifrequency scans of Kirshner et al. ${ }^{2}$ (open squares). The dot-dashed curve shors the result when ${ }^{56}$ i decay is artiflcially suppressed in Model $15 \mathrm{~A}$. 
velocities for the low energ $15 \mathrm{M}_{\theta}$ explosion. The agreement between theory and observation is uniformly within the observational errors, and allows a confident description of the general physical processes which are occurring (s:e also Ref. 13 and 19-22 for conclusions besed on parameterized models).

The initial sharp spike in temperature and luminosity at small photospheric radius corresponds to the breakout of the supernote shock through the surface of the star's supergiant envelope. This is accompanied in theory $7,23-25$ by a +2000 second long, soft $x$-ray pulse with an equivalent black body temperature of $-1.5 \times 30^{5} \mathrm{~K}$ and a peak Iuminosity of $-10^{45} \mathrm{erg} / \mathrm{second}$. The surface is then rapidy cooled by radiative emission and hydrodynamic expansion, balanced in part by radiative diffusion from below.

Time, days

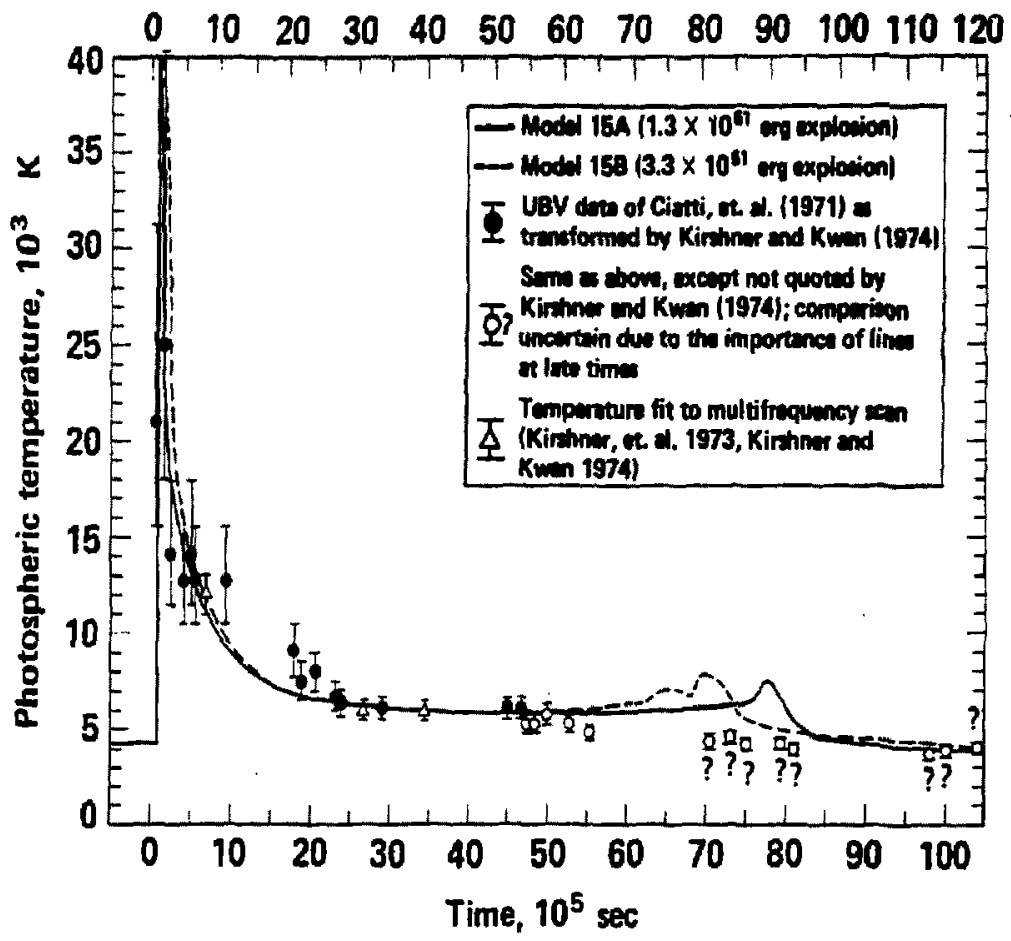

Fig. 6 Photospheric temperature of Type II supernove 1969l compared with $15 \mathrm{M}_{\odot}$ theoretical models. 5 The solid and dotted lines give the results for the $15 \mathrm{M}$ models, while the solid circle data points represent the data of Ciatti, et $2{ }^{16}$ as transformed by Kirshner and Kwan. 18 The open circle data points are obtained by extrapolating the transformation methods of Kirshner and Kwan ${ }^{28}$ to the late time date of Ciatti, et al. ${ }^{16}$, and are associated with "?" marks where lines dominate the spectrum and such color/temperature transformations become dubious. The open triangla data points are derived from fits to multifrequency scans. ${ }^{2}$ 
Time, days

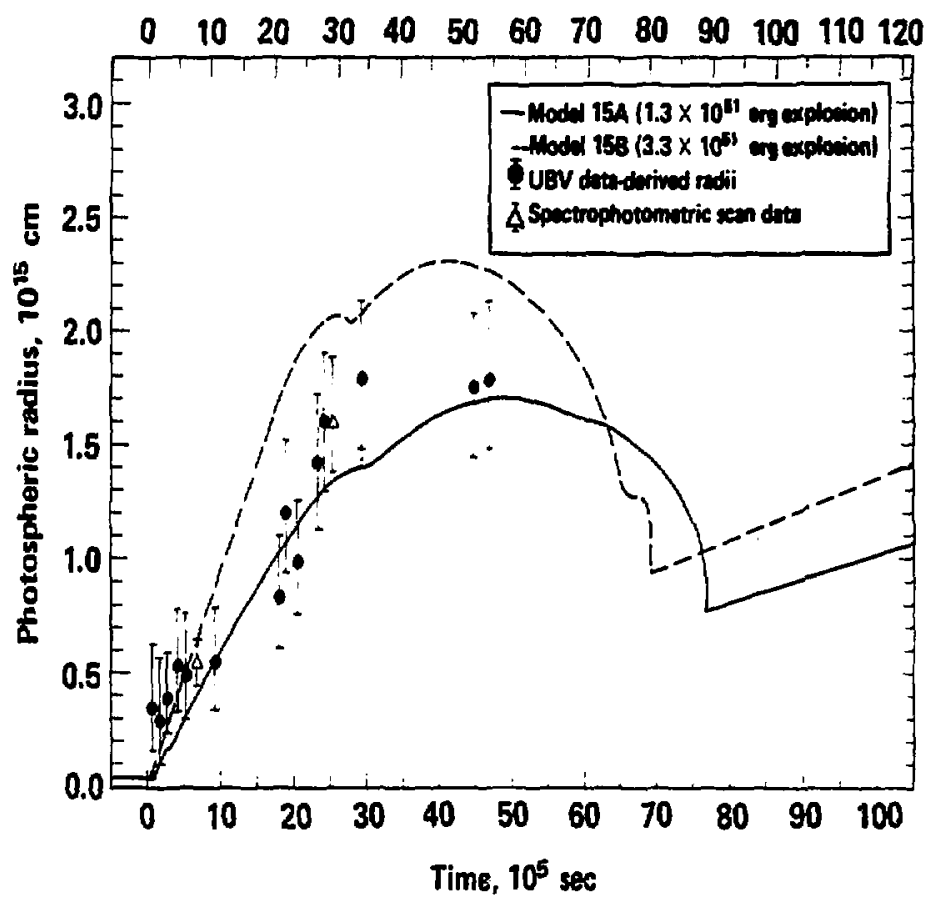

Fig. 7 Photospheric radius of Type II supernova $1969 \mathrm{l}$ compared to $15 \mathrm{M}$ theoretical models. ${ }^{6}$ Solid and dotted lines give the results for the $15 \mathrm{M}$ models, while the $1969 \mathrm{l}$ data points are either derived from UBV data ${ }^{18}$ or multifrequency scans, ${ }^{2}$

The star remains sufficiently optically thick during its acceleration that $99 \%$ of the total supernova energy is converted to kinetic energy of the expanding debris. Only about it thus remains to be radiated when the ster finally starts to become optically thin after expanding to about $10^{15} \mathrm{~cm}$, or about 10 to 30 times its initial radius. As Figure 5 illustrates, this division of energy is just sufficient to explain supernova observations of the SII $1969 \mathrm{k}$ variety. Presupernova stars with radii much larger or much smaller than 3 to $7 \times 10^{13} \mathrm{~cm}$ (corresponding to our 15 and $25 \mathrm{M}_{0}$ models) would respectively radiate too great or too small a fraction of the total supernove energy to agree with the observations.

The photosphere initially lies close to the outer surface of the star, and thus expands rapidly in physical size as the star explodes. Figure 9 shows the evolution of the density profile near the surface of the star during the first 20 days of the explosion, and the position of the photosphere is noted. It is apparent that after the first fer days the density at a given mass coordinate scales as $t^{-3}$ corresponding to an howologous expansion (velocity * radius) with a frozen-out velocity distribution. The photosphere first moves outward 
Time, days

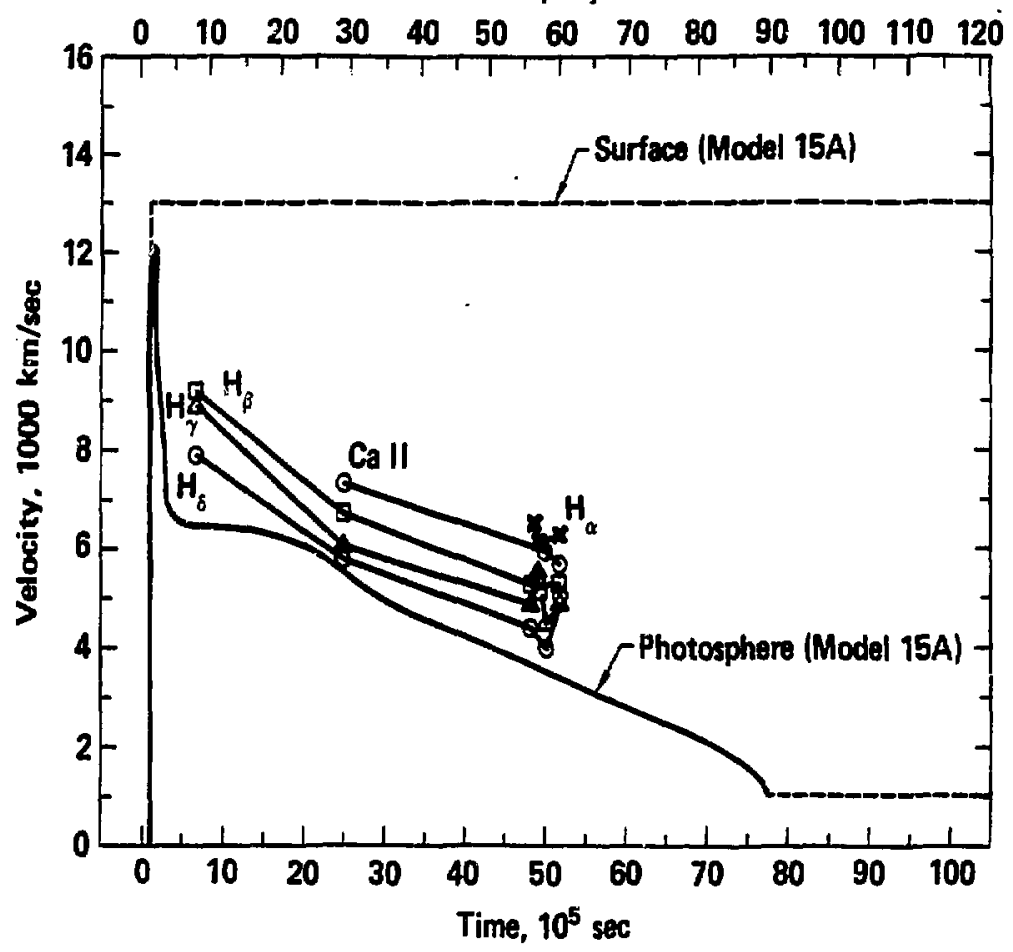

Fig. 8 Time evolution of the surface and photospheric velocities of the $1.3 \times 10^{51} \mathrm{erg}, 15 \mathrm{M}_{0}$ supernova model (15A) compared with absorption lines velocities observed ${ }^{2}$ for supernova $1969 \mathrm{l} .{ }^{6}$

in mass coordinate from its presupernova position due to shockinduced reionization of the overlying material, and then moves inward with respect to the expanding envelope material maintaining an almost constant density of $10^{-13} \mathrm{~g} / \mathrm{cc}$.

After about 20 days, the photosphere has cooled to roughly $6000 \mathrm{~K}$, the temperature at which hydrogen plasma recombines to form a nearly transparent atomic gas at the extremely low densities prevalent in the envelope. This recombination-induced transparency allows the rapid radiative cooling of the immediately underlying layers, causing them in turn to recombine and become transparent. A cooling wave thus develops that sweeps inward through the exploding envelope over a period of about two months. The photosphere follows the recombination front associated with this cooling wave, anit thus its temperature remains close to the $6000 \mathrm{~K}$ recombination temperature (see Fig. 6). In addition, the radius of the photosphere remains nearly constent $\left(=1.5-2.0 \times 10^{15} \mathrm{~cm}\right)$ during this period because the inward motion of the photosphere relative to the envelope material is approximately canceled out by the overall expansion of the 


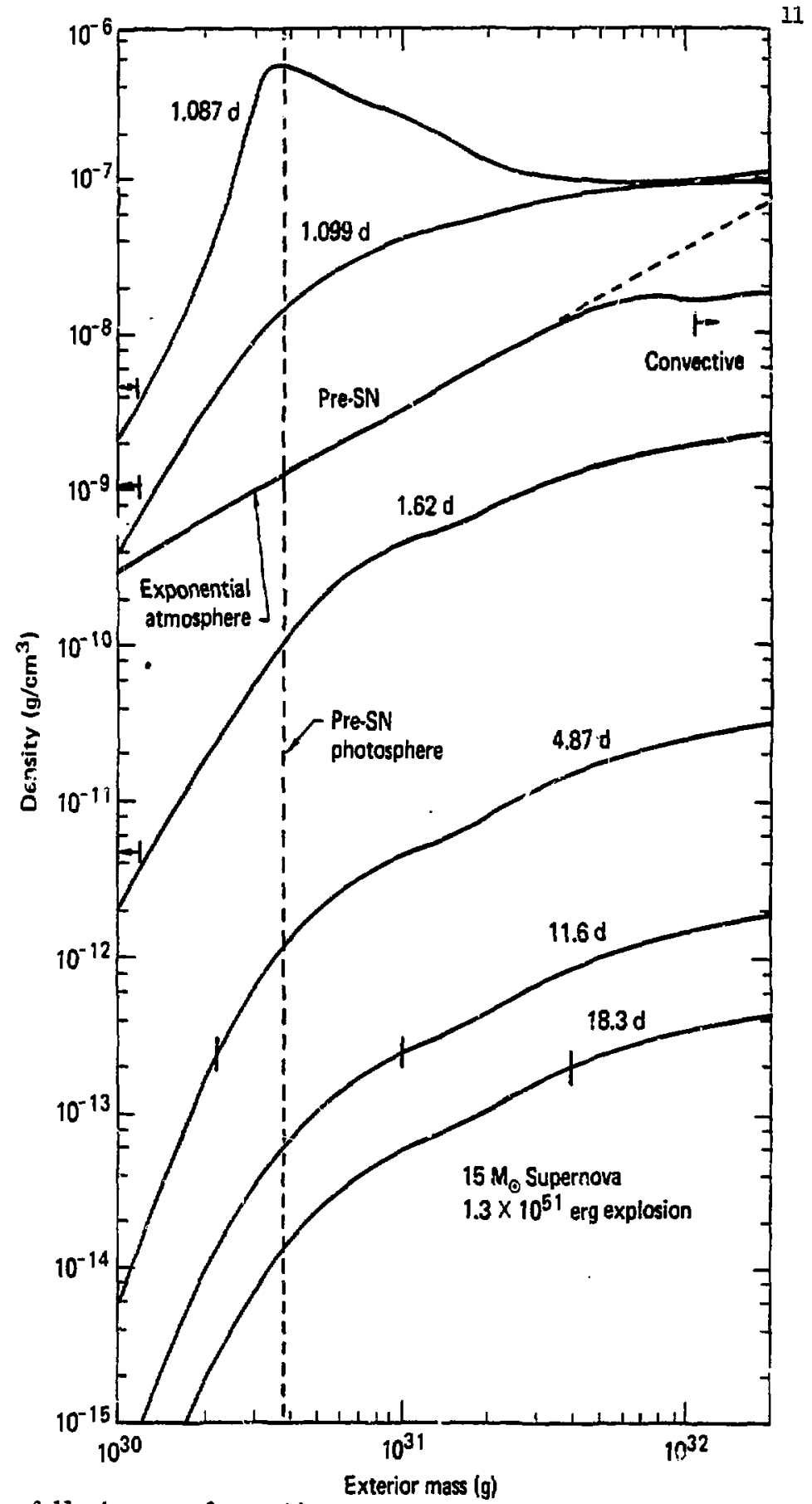

See following page for caption. 
Fig. 9 (previous page) Density profiles as a function of exterior mass coordinate of the $1.3 \times 10^{51} \mathrm{erg}, 15 \mathrm{M}$ supernova model (15A) during the first 20 days of the explosion. The curves are labeled with the time since core collepse, and the position of the photosphere is indisated by a bar. The curve labeled "Pre-SN" is the presupernova density profile, which over most of the mass range shown has the form of an exponential atmosphere (dotted line). As indicated, the presupernova envelope is convective for exterior masses greater than $1.1 \times 10^{32} \mathrm{~g}$.

envelope. It is this combination of nearly constsnt photospheric radius and temperature that causes the supernova's Iuminosity to remain roughly constant during the plateau phase of the light curve (see Fig. 5).

Figures 10 and 11 illustrate the evolution of the temperature and density profiles during this epoch. Note that slight (possibly numericel) irregularities in the nesrly homologous hypersonic flow have induced the growth of density spikes in the mantle.

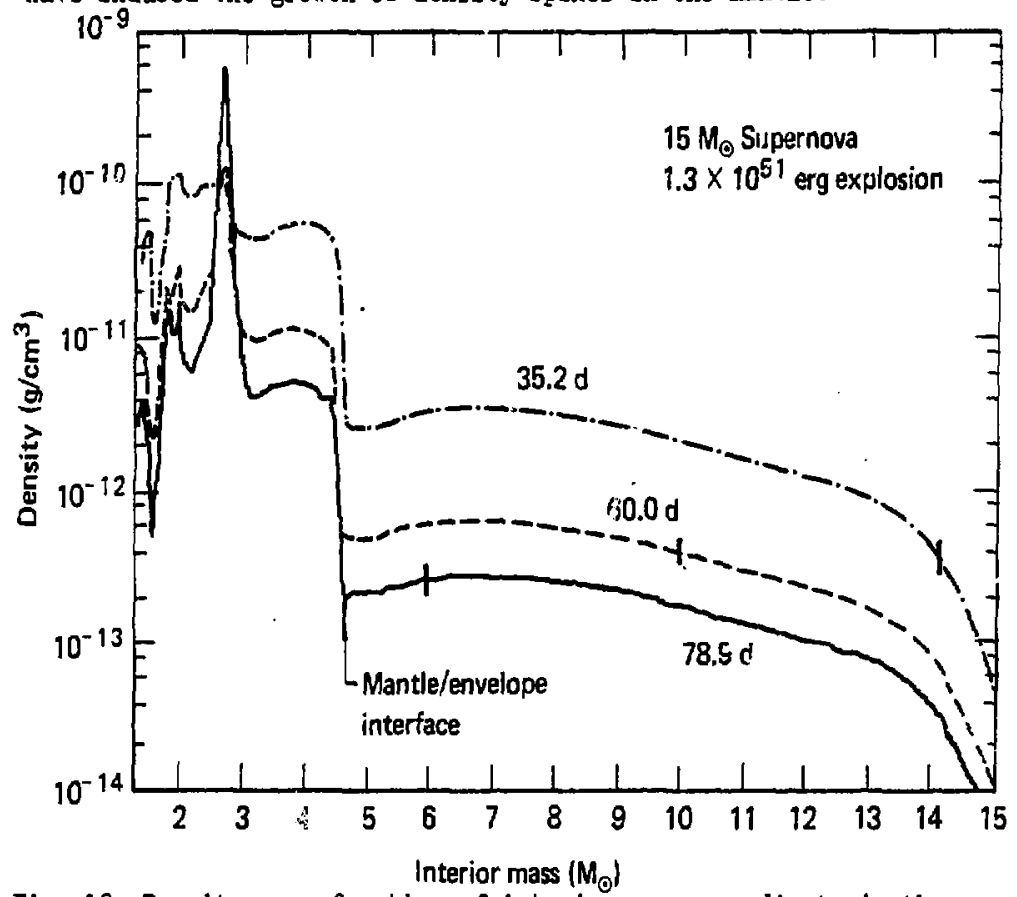

Fig. 10 Density as a runction of interior mass coordinate in the $1.3 \times 10^{51} \mathrm{erg}$, $15 \mathrm{M}$ supernova model during the transparency-waveinduced "plateau" epoch of its light curve. Each curve is labeled with the time since core collapse. The position of the phrtosphere is shown by the ber across each curve. 




Fig. Il Temperature profiles corresponding to the density profiles presented in Fig. 10. Again, the position of the ntosphere is indicated by a bar.

Eventually, as the cooling wave encounters more slowly moving material deep within the envelope, the speed of the wave exceeds the matter velocity, and the photosphere physically shrinks until it encounters the slowly moving $(<1000 \mathrm{~km} / \mathrm{s})$, relatively dense, and very optically thick mantle (see Fig. 7). At this point, a transient increase in the photospheric temperature occurs and persists for approximately two to thr te days as a result of the uncovering and rapid cooling of the hot surface of the mantle. This phenomenon is potentially observable, although the presence of strong emission lines in the overlying envelope may tend to mask it. The sharpness of this final recession and the resulting decrease in luminosity probably are artificially abrupt because of our relatively simplified treatment of recombination and emission-line effects. 5

At late times, the luminosity results from the diffusion out of the mantle of thermalized radiation from the decay of the explosively generated ${ }^{56} \mathrm{Ni}$ and its daughter ${ }^{56} \mathrm{Co}$. As Figure 5 shows, models in which ${ }^{56} \mathrm{Ni}$ decay is turned off display a much more sharply falling luminosity tail as the residual thermal energy in the mantle diffuses out over a characteristic time of only cne to two months. In Ilodels containing energy output from radicactivity, temoorary trapping of the themalized decay energy in the optically thick mantle produces a luminosity decline slower than the 78 -day ${ }^{56} \mathrm{Co}$ half-life, particularly in the $25 \mathrm{M}_{0}$ case. 
The deposition of the positrons and $\gamma$-rays from these decays takes place almost entirely in the mantle due to the large colum density of overlying material that is present even at very late times, as show in Figure 12. As expected, the column density at a given mass coordinate scales as $t^{-2}$ due to the nearly homologous expansion of the star.

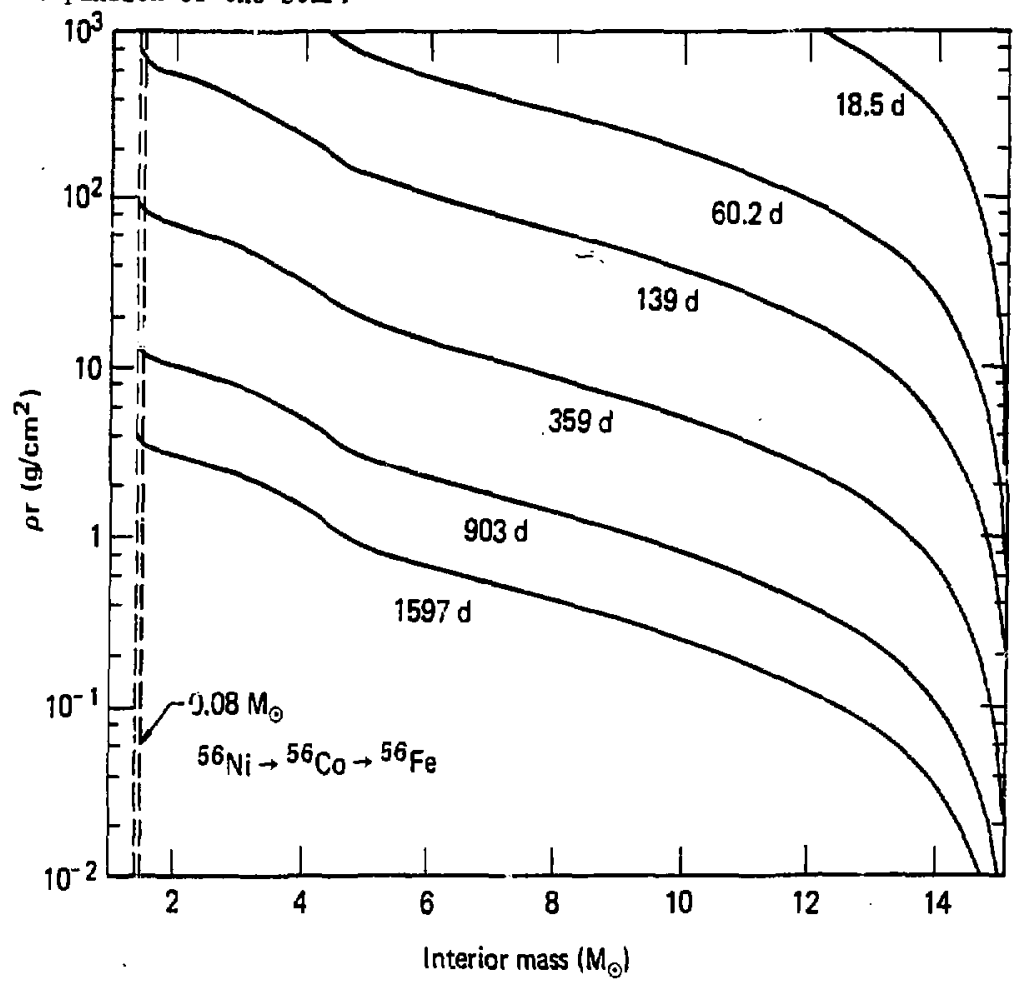

Fig. 12 Colum density, pr, of overlying material as a function of interior mass coordinate in the $1.5 \times 10^{51} \mathrm{erg}, 15 \mathrm{M}$ supernova model. Bach curve is labeled with the time since core collapse, and the position of the $0.08 \mathrm{M}_{0}$ of ${ }^{56} \mathrm{Ni}$ formed in the explosion is indiceted.

The peak emission of unscattered $\gamma$-rays takes place at about 400 days and yields a peak flux in the $3.2 \mathrm{MeV}$ line of about $5 \times 10^{-5}$ photons $\mathrm{cm}^{-2} \mathrm{sec}^{-1} \mathrm{MeV}^{-1}$ (see Ref. 26) for the $1.3 \times 10^{51} \mathrm{erg}, 15 \mathrm{M}_{0}$ supernova model, assuming a distance of I Megaparsec.

The ripples in the theoretical curves at late times result from the formation of density clumps in the mantie noted earlier, which if real appear to offer excellent sites for grain formation. It is likely that two-dimensional instabilities will also occur ${ }^{14}$ (as is suggested by the clumpy appearance of supernova remnants) which should have the effect of increasing the noise, but damping the size of the transient excursions in the luminosity. 
In principal, a great deal of information about supernova can be deduced from their light curves. At times earlier than 3 months, the light curve principally conveys information about the structure of the envelope of the presupernova star. More massive, extended envelopes produce prolonged luminosity plateaus and slower photospheric velocities for fixed shock energies. The behavior of the tail of the light curve, on the other hand, yields information about the size and density of the mantle, and ultimately, its composition.

In order to realize the full potential of these sources of information for understanding supernovae and the elements they produce, it will be necessary to perform detailed calculations of non-LTE supernova atmospheres so that spectral information about composition and plasma conditions can be unfolded. It is hoped that the Type II supernova models presented here will provide a starting point for, and help to motivate such work, as well as extensive new observations of supernovae. Taken together, such advances should allow the confident use of supernovae as both standard 27-28 and "nonstandard candles" $18,21,29$ for determining distances to distant galaxies.

EDGE-LIT CARBON DETONATIONS OF ACCRETING WIITE DWARIS AS TYPE I SUPERNOVAE

Since this workshop is primarily concerned with Type I supernovae, it seems irresistable to broaden the topic of this talk to very briefly describe some of our recent calculations of the fate of white dwarfs undergoing slow mass accretion. i more detailed description will be published elsewhere. 30

Our calculations were started from an initial model supplied by Taam ${ }^{31}$ in which a $0.5 \mathrm{M}_{0}$ white dwarf composed of equal concentrations of carbon and oxygen accretes hycirogen (assumed burned quickly to helium) at a rate of $10^{-8} \mathrm{M}_{\odot} \mathrm{yr}^{-1}$ until $0.62 \mathrm{M}$ of helium has accumulated. The star's density and temperature profiles at this stage are shown in Figure 13. At this point, electron conduction cooling, which has allowed the helium to form a highly degenerate layer on the surface of the $\operatorname{star}\left(p \sim 10^{7} \mathrm{~g} / \mathrm{cc}, \mathrm{T} \sim 5 \times 10^{7} \mathrm{~K}\right)$ is no longer sufficient to counteract the compressional heating of the star due to the increasing overlying mass of helium, and a thermonuclear runaway results at the carbon/helium interface.

Using the stellar evolution/explosion code described above to follow this star's subsequent evolution, we found that, after a brief phase of convective helium burning, the overpressure from the nuclear runaway induces shock waves propagating both outward through the helium layer and inward through the carbon-oxygen core. These shocks ignite further nuclear burning and are rapidly transformed into selfsustaining Chapwan-Jouguet detonation waves. The burning fuel is sufficiently inertially confined so that the final product is predominantly ${ }^{56} \mathrm{Ni}$. The ease with which carbon is ignited in this manner, in contrast to the still unresolved difficulties 32,33 associated with the central ignition of carbon in white drarfs near the Chandraselshar mass, is a direct consequence of the 2-3 order-ofmagnitude reduction in ignition density in the present case which makes possible nuclear-burning-induced overpressures of $400 \%$, instead of $-20-50 \%$. 


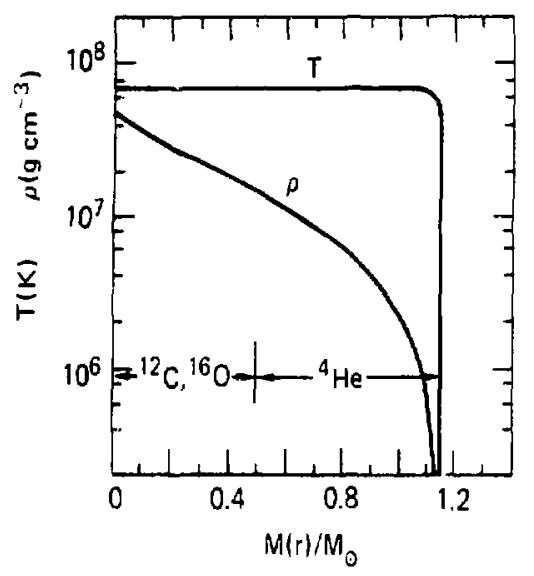

Fig. 13 Density $(0)$ and temperature $(T)$ as a function of interior mess coordinate $\left(M(x) / M_{0}\right)$ in the initial white dwarf model of Tam ${ }^{31}$ just prior to He igmition.

The present status of this calculation is illustrated in Figure It which shows the diverging He and $\mathrm{C} / \mathrm{O}$ nuclear detonation waves just before they reach the surface and center of the star, respectively. Freliminary extensions of this calculation suggest that the light curve and velocities characteristic of Type I supernovae may result as the $=1 M_{0}$ of $6 \mathrm{~N}:$ synthesized by the detonation, and expands and decays.

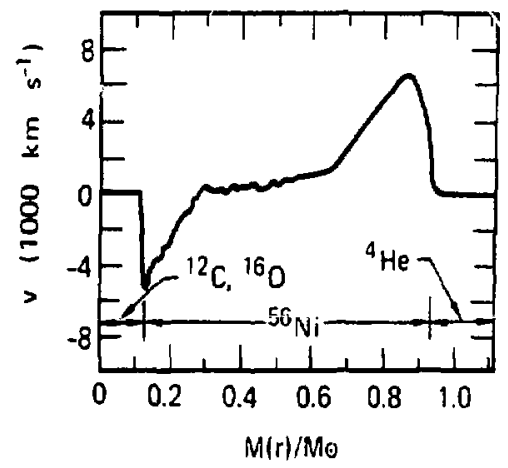

Fig. 14 Velocity and composition profiles of the detonating white dwarf model.

A possible complication is that the He will most likely runeway at a point instead of along an entire spherically symetric mass shell (as we are forced to assume), resulting in a detonation wave propagating spherically outward from a point offset with respect to the center of the star. Ceneric two-dimensional investigations of off-center detonations by Fryxel1 ${ }^{34}$ suggest, however, that provided the detonation can still progagate against the initially higher rate of geometric divergence, the final state of the detonated star should be nearly the same. 
Ron Taan is gratefuily acknowledged for supplying details of his accreting white dware models prior to publication and for useful discussions.

\section{REFERENCES}

1. R. Minkowski, Annual Reviews, 2, 247 (1964).

?. R. P. Kirshner, J. B. Oke, M. V. Penston, and I. Searle, Ap. J. 185,303 (1973) and references cited therein; R. P. Kirshner, and J. B. Oke, Ap. J. 200, 574 (1975).

3. I. S. Shiklovsky, Supernovee (Wiley, New York, 1968), p. 20.

4. J. Maza and S. van den Bergh 204, 519 (1976).

5. T. A. Weever, G. B. Zimerman, and S. E. Woosley, Ap. J. 225, 1021 (1978). Also see references therein.

6. T. A. Weaver and S. E. Woosley, Proc. 9tin Texas Symposium on Relativistic Astrophysics, Munich, 1978, Ann. N.Y. Acad. Sci. 336, 335 (1980).

7. T. A. Weaver and S. E. Woosley (1980), in preparation.

8. S. E. Woosley and T. A. Weaver $(1980)$, in preparation.

9. E. M. Burbidge, G. R. Burbidge, W. A. Fowler, and F. Hoyle, Rev. Mod. Phys. 29, 547 (1957).

10. J. R. Wilson and R. L. Bowers (1978), private comunication; J. R. Wilson, Proc. 9th Texas Symposium on Relativistic Astrophysics, Munich, 1978, Ann. N.Y. Acad. Sci. 336, 358, (1980).

11. Ya. B. Zel'dovich and Yu. P. Raizer, Physics of Shock Waves and High-Temperature Hydrodynamic Phenomena (Academic, New York, 1966), p. 93.

12. G. E. Miller and J. M. Scalo, Ap. J. Suppl. 41, 513 (1979).

13. R. A. Chevalier, Ap. J. 207, 872 (1976).

14. R. A. Chevalier and R. I. Klein, Ap. J. 219, 931 (1978).

15. G. J. Lasher, Ap. J. 201, 194 (1975), and this conference.

16. F. L. Ciatti, L. Rosino, and F. Bertola, Mem. Soc. Astron. Ital. $42,163(1971)$.

17. R. Barbon, F. Ciatti, and L. Rosino, Astron, \& Astrophys. 29, 57 (2973).

19. R. P. Kirshner and J. Kwan, Ap. J. 193, 27 (1974).

19. S. W. Falk and W. D. Arnett, Ap. J. Lett. 180, L65 (1973);

S. W. Falk and W. D. Arnett, Ap. J. Suppl. 33, 515 (1976).

20. W. D. Arnett and S. W. Falk, Ap. J. 210, 733 (1976).

21. S. R. Schurmann, W. D. Arnett, and S. W. Falk, AD. J. 230, 11 (1979).

22. E. K. Grassberg, V. S. Imshennick, and D. K. Nadyozhin, Astrophys. Space Sci. 10, 28 (1971).

23. G. J. Lasher and K. I. Chan, Ap. J. 230, 742 (1979).

24. R. I. Klein and R. A. Chevalier, Ap. J. Iett. 223, L109 (1978).

25. R. A. Chevalier and R. I. Klein, Ap. J. 234, 597 (1979).

26. T. S. Axelrod, private communication (1980).

27. L. Rosino, in Supernovae, ed. D. N. Schramm (Reidel, DordrechtHollend, 1977), p. 1.

28. L. Rosino and G. Di Tullio, in Supernovae and Supernova Remnants, ed. C. B. Cosmovici (Reidel, Dordrecht-Holland, 1974), p. 19. 
29. D. Branch, in Supernovae, ed. D. N. Schramm (Reidel, DordrechtHolland, 1977), p. 21; R. V. Hagoner, Ap. J. Lett. 214, L5 (1977).

30. S. E. Woosley, T. A. Weaver, and R. E. Taam (1980), in preparation.

31. R. E. Taam, Ap. J. 237, 142 (1980) and referernes thersin; ind private communication (1979).

32. R. G. Couch and W. D. Arnett, Ap. J. 196, 791 (1975); S. W. Bruenn and A. Marroguin 195, 567 (1975) and references therein.

33. J. C. Wheeler, J-R. Buchler, and Z. K. Barkat, 184, 897 (1973); D. Sugimoto and K. Nomoto, preprint (1979), and references cited therein.

34. B. A. Fryxel1, Ap. J. 234, 641 (1979). 